\title{
A BOUND FOR ENTIRE HARMONIC FUNCTIONS OF THREE VARIABLES*
}

\author{
By CHI YEUNG LO, Michigan State University
}

In [6], Professor R. P. Gilbert has obtained an upper bound and a lower bound for an entire GASPT ${ }^{1}$ function by the integral operator method. Similar methods can be applied to estimate bounds for harmonic functions of three variables $x_{1}, x_{2}, x_{3}$.

Definition. Let $g(u, \zeta)$ be an analytic function defined by

$$
g(u, \zeta)=\sum_{n=0}^{\infty} \sum_{m=-n}^{n} a_{n, m} u^{-n-1} \zeta^{m}
$$

in some region in the space $C^{2}$ (space of two complex variables). $g(u, \zeta)$ is said to belong to the class $K$ if $g(u, \zeta)$ is entire in the $u^{-1}$ plane and the variable $\zeta$ is restricted to some compact subset in the $\zeta$-plane.

Let $g(u, \zeta)$ be defined on $B(R, \delta) \equiv\{|u| \geq R\} \times\{1-\delta \leq|\zeta| \leq 1+\delta\}$. Then the three dimensional harmonic functions $u\left(x_{1}, x_{2}, x_{3}\right)=U(r, \theta, \phi)$ regular at infinity can be generated by the Whittaker-Bergman operator, written as

$$
U(r, \theta, \phi)=\mathbf{B}_{3}[g] \equiv \frac{1}{2 \pi i} \int_{|\zeta|=1} g(u, \zeta) \frac{d \zeta}{\zeta}
$$

where

$$
U(r, \theta, \phi)=\sum_{n=0}^{\infty} \sum_{m=-n}^{n} \frac{a_{n, m}(n-m) !}{n !}(-i)^{m} r^{-n-1} P_{n}^{m}(\cos \theta) e^{i m \phi}
$$

and

$$
u=r\left\{\cos \theta+i \sin \theta\left(e^{i \phi} / \zeta+\zeta / e^{i \phi}\right)\right\} .
$$

If we analytically continue $x_{1}, x_{2}, x_{3}$ into the complex number space, and introduce the complex spherical variables

$$
r=+\left(x_{1}^{2}+x_{2}^{2}+x_{3}^{2}\right)^{1 / 2}, \quad \eta=+\left(x_{1}+i x_{2} / x_{1}-i x_{2}\right)^{1 / 2}, \quad \text { and } \xi=x_{3} / r
$$

then an inverse operator $\mathrm{B}_{3}^{-1}[U]$ can be constructed as

$$
g(s, \zeta)=\mathbf{B}_{3}^{-1}[U] \equiv \frac{1}{4 \pi i} \int_{-1}^{1} d \xi \int_{|\eta|-1} \frac{r(s+u)}{(s-u)^{2}} U(r, \xi, \eta) \frac{d \eta}{\eta}
$$

where $u=r\left[\xi+(i / 2)\left(1-\xi^{2}\right)^{1 / 2}(\zeta / \eta+\eta / \zeta)\right]$ and $U(r, \xi, \eta)$ is analytic in the polycylinder

$$
\begin{aligned}
& D\left(\epsilon_{1}, \epsilon_{2}, \epsilon_{3}\right) \\
& \quad \equiv\left\{\left.(r, \xi, \eta)|| r\right|^{2} \geq \frac{1}{2 \epsilon_{1}} ;|1-\xi|+|1+\xi| \leq 2+\epsilon_{2}, 1-\epsilon_{3} \leq|\eta| \leq 1+\epsilon_{3}\right\} .
\end{aligned}
$$

For details of the construction of these operators, please refer to [5].

*Received March 6, 1967; revised manuscript received August 7, 1967.

${ }^{1}$ Generalized axially symmetric potential theory. 
Definition. We shall say $H(r, \xi, \eta)$ belongs to the class $E$ in $C^{3}$ (space of three complex variables) if $H(r, \xi, \eta)$ is entire in the $r^{-1}$ plane while the other variables $\xi, \eta$ are confined to some compact subsets in their respective planes.

Remark. If $x_{1}, x_{2}, x_{3}$ are real, then $\xi=\cos \theta, \eta=e^{\phi i}$, and $U(r, \xi, \eta)=U(r, \theta, \phi)$. We shall say $U(r, \theta, \phi)$ belongs to the class $E_{R}$ in the $R^{3}$ space if $U(r, \theta, \phi)$ is entire in the variable $r^{-1}$ while $-\pi \leq \theta \leq \pi$ and $0 \leq \phi \leq 2 \pi$, i.e., $U(r, \theta, \phi)$ is an entire harmonic function regular at infinity in the $R^{3}$ space.

By the maximum principle for two complex variables, $|g(u, \zeta)|$ attains its maximum on the Bergman-Silov boundary of $B(R, \delta)$, and is denoted by $M_{v}(R, \delta)$. By elementary estimates, $|u| \leq\left(1+\epsilon^{\prime}\right)|r|$ for sufficiently small $\epsilon^{\prime}$, then we have, from (1),

$$
|U(r, \theta, \phi)| \leq M_{0}(R, \delta)
$$

which holds for $|r| \geq R /\left(1+\epsilon^{\prime}\right)$.

This result can be extended to the case when $g(u, \zeta)$ is in the class $K$. Since all the terms of $g(u, \zeta)$ involve only the negative powers of $u$, we are interested in investigating the asymptotical behavior of $g(u, \zeta)$ when $R \rightarrow 0$ by restricting $\zeta$ in the compact set $\{\zeta|1-\delta \leq| \zeta \mid \leq 1+\delta\}$.

Suppose $M_{0}(R, \delta)$ has the order of growth $\lambda, 0<\lambda<\infty$, with respect to $1 / R$ for any fixed but arbitrary $\delta$, then we have asymptotically,

$$
\exp \left(R^{-\lambda+\epsilon}\right)<M_{\theta}(R, \delta)<\exp \left(R e^{-\lambda-\epsilon}\right)
$$

for every $\epsilon>0$.

It follows from (3) that

$$
\left|M_{U}\left(R, \epsilon_{2}, \epsilon_{3}\right)\right|<\exp \left(R^{-\lambda-\epsilon}\right)
$$

where $M_{U}\left(R, \epsilon_{2}, \epsilon_{3}\right)=\sup |U(r, \theta, \phi)|,(r, \theta, \phi) \varepsilon D\left(\epsilon_{1}, \epsilon_{2}, \epsilon_{3}\right)$ with $\epsilon_{1}=\left(1+\epsilon^{\prime}\right) / 2 R^{2}$.

In order to obtain a lower bound for $M_{U}\left(R, \epsilon_{2}, \epsilon_{3}\right)$, we consider the inverse operator $\mathbf{B}_{3}^{-1}[U]$. From properties concerning Cauchy integrals of analytic function of several complex variables, [4, p. 53], both $U(r, \xi, \eta)$ and $g(s, \zeta)$ are analytic if their kernels $g(u, \zeta)$ and $\left(r(s+u) /(s-u)^{2}\right) U(r, \xi, \eta)$ are analytic in their respective domains of definition, and their paths of integration are piecewise smooth curves. It is therefore easy to observe that if $g(u, \zeta)$ is in the class $K$, then $U(r, \xi, \eta)$ is in the class $E$. The converse is also true provided that we can confine the variables $s, u$ in suitable domains such that the function $m(r, s, u)=r(s+u) /(s-u)^{2}$ remains bounded.

Let $|r|=R$ and $|s|=2 R=R_{1}$. Then

$$
|m(r, s, u)|=\left|\frac{r(s+u)}{(s-u)^{2}}\right| \leq \frac{|r|\{|s|+|u|\}}{\{|s|-|u|\}^{2}} \leq \frac{R\left\{2 R+\left(1+\epsilon^{\prime}\right) R\right\}}{R^{2}\left(2-1-\epsilon^{\prime}\right)} \leq \frac{3+\epsilon^{\prime}}{1-\epsilon^{\prime}}
$$

For sufficiently small $\epsilon^{\prime}$, say $0<\epsilon^{\prime}<1 / 3,|m(r, s, u)|<5$.

Let $x_{1}, x_{2}, x_{3}$ be real, then $U(r, \xi, \eta)=U(r, \theta, \phi)$. If $U(r, \theta, \phi)$ is in the class $l_{R}$, then $g(s, \zeta)$ is in the class $K$; an estimate for a lower bound of $U(r, \theta, \phi)$ shows that

$$
\exp \left(R_{1}^{-\lambda+\epsilon}\right)<M_{\vartheta}\left(R_{1}, \delta\right)<5 M_{U}\left(R, \epsilon_{2}, \epsilon_{3}\right) .
$$

Let

$$
\exp \left(R^{-\lambda+\tilde{\epsilon}}\right)=\exp \left(R_{1}^{-\lambda+\epsilon}\right) / 5<M_{U}\left(R, \epsilon_{2}, \epsilon_{3}\right)
$$


Hence,

$$
\begin{gathered}
R^{-\lambda+\dot{\epsilon}}=R_{1}^{-\lambda+\epsilon}-\log 5 \\
R^{-\lambda+\tilde{\epsilon}} / R_{1}^{-\lambda+\epsilon}=1-(\log 5) /\left(R_{1}^{-\lambda+\epsilon}\right)=1-R_{1}^{\lambda-\epsilon} \log 5 .
\end{gathered}
$$

As $R_{1} \rightarrow 0$, we have $R^{-\lambda+i} / R_{1}^{-\lambda+\epsilon} \approx 1$

$$
\begin{aligned}
R^{-\lambda+\tilde{\epsilon}} & \approx R_{1}^{-\lambda+\epsilon}=2^{-\lambda+\epsilon} R^{-\lambda+\epsilon} \\
R^{\tilde{\epsilon}} & \approx 2^{-\lambda+\epsilon} R^{\epsilon} \\
\bar{\epsilon} \log R & \approx(-\lambda+\epsilon) \log 2+\epsilon \log R \\
\tilde{\epsilon} & \approx \epsilon+(-\lambda+\epsilon) \log 2 / \log R .
\end{aligned}
$$

As $R \rightarrow 0$, we have $\bar{\epsilon} \approx \epsilon$. From (7), we get

$$
\exp \left(R^{-\lambda+\epsilon}\right)<M_{U}\left(R, \epsilon_{2}, \epsilon_{3}\right) \text {. }
$$

Combining the inequalities (5) and (8) we have proved:

Theorem 1. Let $U(r, \theta, \phi)$ be an entire harmonic function, regular at infinity, in $R_{3}$-space. Then there exists a number $\lambda$ (its order) such that for $R$ sufficiently small and $\epsilon>0$, the maximum modulus $M_{U}\left(R, \epsilon_{2}, \epsilon_{3}\right)$ for $U(r, \theta, \phi)$ is bounded by

$$
\exp \left(R^{-\lambda+\epsilon}\right)<M_{U}\left(R, \epsilon_{2}, \epsilon_{3}\right)<\exp \left(R^{-\lambda-\epsilon}\right) .
$$

Theorem 1 implies the function $U(r, \theta, \phi)$ has the same order as its entire associate $g(u, \zeta)$, and vice versa.

A formula on the type of an entire function $U(r, \theta, \phi)$ of order $\lambda$ can be derived from the corresponding formula of its associate function $g(u, \zeta)$ from (1).

Theorem 2. Let $U(r, \theta, \phi)$ be an entire harmonic function regular at infinity of order $\lambda$. Then the type $\alpha$ of

$$
U(r, \theta, \phi)=\sum_{n=0}^{\infty} \sum_{m=-n}^{n} a_{n, m} r^{-n-1} P_{n}^{m}(\cos \theta) e^{i m \phi}
$$

is given by the formula

$$
\frac{e}{(1-\delta)}(e \alpha \lambda)^{1 / \lambda}=\lim _{n \rightarrow \infty} \sup n(n+1)^{1 / \lambda}\left|a_{n n}\right|^{1 /(n+1)}
$$

where $g(u, \zeta)$, the associate of $U(r, \theta, \phi)$ in $(1)$, has $B(R, \delta)$ as its domain of definition.

Proof. The associate function $g(u, \zeta)$ is defined by

$$
g(u, \zeta)=\sum_{n=0}^{\infty} \sum_{m=-n}^{n} a_{n, m} \frac{n !}{(n-m) !}(-i)^{m} \zeta^{m} u^{-n-1}=\sum_{n=0}^{\infty} P_{n}(\zeta) u^{-n-1}
$$

where

$$
P_{n}(\zeta)=\sum_{m=-n}^{n} b_{n, m} \zeta^{m}=\sum_{m=-n}^{n} a_{n, m} \frac{n !}{(n-m) !}(-i)^{m} \zeta^{m} .
$$

Let $M_{P_{n}}(\delta)=\sup _{1-\delta \leq|\zeta| \leq 1+\delta}\left|P_{n}(\zeta)\right|$. Considering the function $g(u, \zeta)$ as a function of the variable $u$ alone, and applying Cauchy's inequality, we obtain

$$
M_{P_{n}}(\delta) \leq M_{o}(R, \delta) R^{n+1} .
$$


By our previous remark, $g(u, \zeta)$ is entire with respect to $1 / u$, of order $\lambda$ and type $\alpha$, say. Then

$$
M_{P_{n}}(\delta) \leq \exp \left(\alpha R^{-\lambda}\right) R^{n+1} \quad \text { for all } R \text { when } R \rightarrow 0 .
$$

The minimum value of $R$ is obtained when $R=(\alpha \lambda /(n+1))^{1 / \lambda}$. Hence,

$$
M_{P_{n}}(\delta) \leq(e \alpha \lambda /(n+1))^{(n+1) / \lambda} .
$$

$P_{n}(\zeta)$ is analytic in the annulus $1-\delta \leq|\zeta| \leq 1+\delta$. Using the representation formula for the Laurent coefficients for $P_{n}(\zeta)$, we have

$$
b_{n, m}=\frac{1}{2 \pi i} \int_{|\zeta|-\rho} \frac{P_{n}(\zeta) d \zeta}{\zeta^{m+1}}, \quad 1-\delta \leq \rho \leq 1+\delta .
$$

Then

$$
\left|b_{n, m}\right| \leq M_{P_{n}}(\delta) \rho^{-m} .
$$

In order to estimate $\left|b_{n, m}\right|$ for different $m$ and a fixed $n$, we consider two cases:

(i) $m \geq 0$, the maximum bound $M_{1}$ for $\left|b_{n, m}\right|$ occurs at $\rho=1-\delta, m=n$. Hence $M_{1}=M_{P_{\mathrm{a}}}(\delta) /(1-\delta)^{n}$.

(ii) $m<0$, the maximum bound $M_{2}$ for $\left|b_{n, m}\right|$ attains when $\rho=1+\delta, m=-n$. Hence, $M_{2}=M_{P n(\delta)}(1+\delta)$, but

$$
\begin{aligned}
M_{1}-M_{2} & =M_{P_{n}(\delta)}\left[(1-\delta)^{-n}-(1+\delta)^{n}\right] \\
& =M_{P_{n}(\delta)} \delta^{2}\left[1+\left(1-\delta^{2}\right)+\cdots\right](1-\delta)^{-n} \geq 0 .
\end{aligned}
$$

For each $n, m=-n,-n+1, \cdots, n-1, n$, the maximum bound for $b_{n, m}$ occurs when $m=n$ and $R_{2}=1-\delta$. Hence,

$$
\left|b_{n, n}\right|=\max \left|b_{n, m}\right| \leq M_{P_{n}(\delta)}(1-\delta)^{-n} \leq(e \alpha \lambda /(n+1))^{(n+1) / \lambda}(1 /(1-\delta))^{n+1} .
$$

Select a subseries $g(u, \zeta)=\sum_{n=0}^{\infty} b_{n, n} \zeta^{n} u^{-n-1}$ of $g(u, \zeta)$. We have

$$
\limsup _{n \rightarrow \infty}\left|b_{n, n}\right| \geq \limsup _{m+n \rightarrow \infty}\left|b_{n, m}\right| \text {. }
$$

We can derive the formula for the type of $g(u, \zeta)$, considered as an entire function of one complex variable in $1 / u$, as

$$
\limsup _{n \rightarrow \infty}(n+1)^{1 / \lambda}\left|b_{n, n}\right|^{1 /(n+1)}=\frac{1}{1-\delta}(e \alpha \lambda)^{1 / \lambda} \quad \text { see [3, p.292]. }
$$

This implies

$$
\begin{aligned}
\frac{(e \alpha \lambda)^{1 / \lambda}}{(1-\delta)} & =\limsup _{n \rightarrow \infty}(n+1)^{1 / \lambda}\left|b_{n, n}\right|^{1 /(n+1)}=\limsup _{n \rightarrow \infty}(n+1)^{1 / \lambda}\left|a_{n, n}\right|^{1 /(n+1)}|n !|^{1 /(n+1)} \\
& =\limsup _{n \rightarrow \infty} \frac{n}{e}(n+1)^{1 / \lambda}\left|a_{n, n}\right|^{1 /(n+1)} .
\end{aligned}
$$

This completes the proof. 


\section{REFERENCES}

[1] S. Bergman, Some properties of a harmonic function of three variables given by its series development, Arch. Rational Mech. Anal. 8, 207-222 (1961)

[2] S. Bergman, Sur les singularités des fonctions harmoniques de trois variables, Comptes rendus des seances de l'Academie des Sciences 254, 3482-3483 (1962)

[3] P. Dienes, The Taylor series, Dover, New York, 1957

[4] B. A. Fuks, Introduction to the theory of analytic functions of several complex variables, Transl. Math. Monographs, Vol. 8, Amer. Math. Soc., Providence, R. I., 1963

[5] R. P. Gilbert, Singularities of three dimensional harmonic functions, Pacific J. Math 10, 1243-1255 (1961)

[6] R. P. Gilbert, Some inequalities for generalized axially symmetric potentials with entire and meromorphic associates, Duke Math. J. 32, 239-245 (1965) 